TẠP CHÍ KHOA HỌC ĐẠI HỌC TÂN TRÀO
ISSN: 2354 - 1431

\title{
Góp phần nghiên cứu về thành phần hóa học của cây Sưa đỏ (dalbergia tonkinesis) ở Tây Nguyên
}

\author{
Ngũ Truò̀ng Nhân ${ }^{a^{*}}$, Nguyễn Mạnh Cường ${ }^{b}$ \\ ${ }^{a}$ Trưòng Đại học Tây Nguyên \\ ${ }^{b}$ Viện Hàn Lâm Khoa học và Công nghệ Việt Nam \\ *Email:ntnhan@ttn.edu.vn
}

\section{Thông tin bài viết}

Ngày nhận bài:

16/02/2020

Ngày duyệt đăng:

10/3/2020

Tù khoá:

Sua đó; chi Trắc;

Isoflavanone; sativanone;

phân lập

\section{Tóm tắt}

Từ gỗ cây Sưa đỏ (Dalbergia tokinensis Prain) thu hái tại tỉnh Dak Lak. Sử dụng kết hợp các phương pháp sắc ký bản mỏng và sắc ký cột. Hợp chất có khung isoflavanone là sativanone được phân lập. Cấu trúc hóa học của hợp chất này được xác định bằng phổ cộng hường từ hạt nhân NMR (phổ $1 \mathrm{H}-\mathrm{NMR}$, 13C-; DEPT-NMR), đồng thời đối chiếu với các tài liệu tham khảo đã công bố.

\section{Mở đầu}

Tính đến nay, chi Trắc (Dalbergia L.f.) có khoảng 647 loài, trong đó 304 loài đã được xác nhận. Ở Việt Nam hiện đã thống kê được khoảng 27 loài [1].

Một số loài thuộc chi này có giá trị cao về gỗ như: Sưa (Dalbergia tonkinensis Prain), Trắc (Dalbergia cochinchinensis), Hồng sắc Ấn Độ (Dalbergia latifolia) và Cẩm lai (Dalbergia oliveri) [2]. Trong đó, hai loài Trắc và Sưa đỏ bị khai thác quá mức, hiện có trong danh mục sách Đỏ Việt Nam (2007), phân hạng ở mức nguy cấp (EN) [3].

Báo cáo về hóa học chỉ ra các loài thuộc chi Trắc sở hữu phong phú các khung chất: flavonoid, isoflavonoid, xanthone, quinone, terpenoid, chalcone, aurone,..., rất nhiều hợp chất sở hữu nhiều tác dụng sinh học quí như: ho suyễn, tiểu đường, tim mạch, v/v [4-6]. Những kết quả bước đầu về khía cạnh hóa học, sinh học và thực vật của loài Sưa đỏ (Dalbergia tonkinensis Prain) đã được công bố [6-8]. Trong khuôn khổ bài báo này. Chúng tôi mô tả kết quả chiết tách, phân lập và xác định cấu trúc hợp chất sativanone, lần đầu tiên được tìm thấy ở loài Sưa (Dalbergia tonkinensis Prain).

\section{Nội dung và phương pháp nghiên cứu \\ 2.1. Đối tự̂ng nghiên cưu}

Cây sưa đỏ (Dalbergia tonkinensis Prain) thu ở Dak Lak.

\subsection{Phương pháp nghiên cúu}

Sử dụng các phương pháp tiếp cận thường qui về hóa học thực vật.

\subsection{Thưc nghiệm}

\subsubsection{Thiết bị và dụng cu}

Phổ cộng hưởng từ hạt nhân được ghi trên máy Bruker Avance $500 \mathrm{MHz}$ tại Viện Hoá học, Viện Hàn lâm Khoa học và Công nghệ Việt Nam và TMS được sử dụng làm chất nội chuẩn. Sắc ký lớp mỏng (TLC) được thực hiện trên bản mỏng Silica gel Merck $60 \mathrm{~F}_{254}$, $\mathrm{RP}_{18} \mathrm{~F}_{254 \mathrm{~s}}$. Sắc ký cột $(\mathrm{CC})$ được thực hiện trên chất hấp phụ là Silica gel (Merck) cõ hạt 0,040-0,063 mm (Merck) và cột sắc ký pha đảo RP-18 (Merck), đèn UV hai chùm tia ở các bước sóng $254 \mathrm{~nm}$ và $365 \mathrm{~nm}$.

\subsubsection{Hóa chất}


Các hóa chất sử dụng trong thí nghiệm đều đảm bảo độ tinh khiết bao gồm các dung môi methanol, chlorofom, aceton, ethyl acetate, nước cất.

Thuốc thử $\mathrm{H}_{2} \mathrm{~S}_{4}(10 \%), \mathrm{FeCl}_{3} / \mathrm{HCl}$ dùng để phát hiện các hợp chất phenol và dẫn xuất.

\section{Kết quả và thảo luận}

\subsection{Kết quả dũ liệu phổ}

Cặn dịch chiết chloroform (15,1 g, MC) được hoà tan bằng một lượng methanol vừa đủ. Dung dịch thu được đem trộn với một lượng silicagel Merck (1/1) làm khô, nghiền thành dạng bột mịn. Bột silicagel có chứa dịch chiết này được đưa lên cột sắc ký đường kính $2,5 \mathrm{~cm}$, được nhồi 250 g silicagel Merck (loại 400-630 mesh) theo phương pháp nhồi cột ướt. Cột sau khi đã tương đối ổn định, bột silicagel chứa dịch chiết được đưa lên đầu cột với hệ gradient dung môi rửa giải là chloroform/ethyl acetate (10/1-5/2) tăng dần độ phân cực thu được 6 phân đoạn từ $\left(\mathrm{C}_{1}-\mathrm{C}_{6}\right)$. Phân đoạn $\mathrm{C}_{2}(1,5 \mathrm{~g})$ được tiếp tục phân tách trên cột $\mathrm{CC}$, silicagel pha thường với hệ dung môi rửa giải chloroform/ethyl acetate $(5 / 2,2 / 1, \mathrm{vv})$ thu được 5 phân đoạn được ký hiệu từ $\left(\mathrm{C}_{2.1}-\mathrm{C}_{2.5}\right)$. Phân đoạn $\mathrm{C}_{2}$ được rửa giải bởi 5 hệ dung môi: Phân đoạn $\mathrm{C}_{2.1}$ thu được một mẫu sạch đem chấm bản mỏng và soi trên đèn UV thấy được 1 chấm tròn (mẫu được giải ly với 4 hệ dung môi khác nhau: C:A, C:E, C:E:M, H:M), sau đó mẫu được làm khô trong máy sấy ở nhiệt độ $60^{\circ} \mathrm{C}$ thu được hợp chất sạch (1) (20mg).
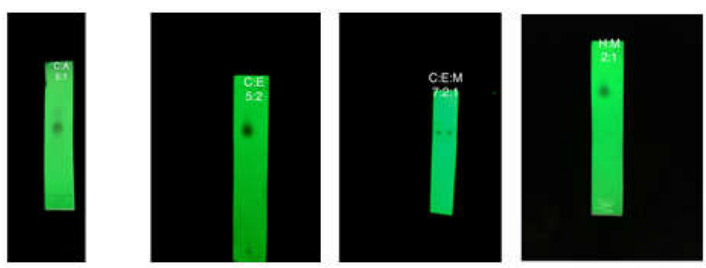

Hình 1. Bản mỏng (1) soi trên đèn UV ở bước sóng $254 \mathrm{~nm}$
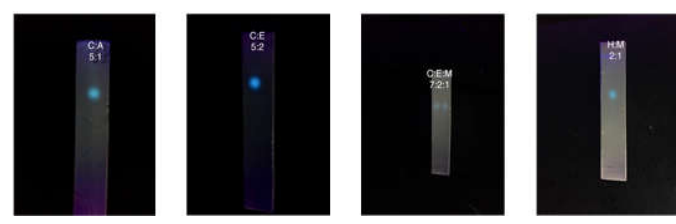

Hình 2. Bản mỏng (1) soi trên đèn UV ở bước sóng $365 \mathrm{~nm}$

Dữ kiện phổ (1): ${ }^{1} \mathrm{H}-\mathrm{NMR}\left(500 \mathrm{MHz}, \mathrm{DMSO}-\mathrm{d}_{6}\right)$ : 7,67 (1H, d, 9,0, H-5), 6,97 (1H, d, 8,5, H-6'), 6,57 (1H, d, 2,0, H-8), 6,52 (1H, dd, 9,0, 2,5, H-6), 6,46 (1H, dd, 8,5, 2,5, H-5'), 6,34 (1H, d, 2,0, H-3'), 4,52 (1H, t, 11,0, $\left.\mathrm{H}_{\mathrm{a}}-2\right), 4,40\left(1 \mathrm{H}, \mathrm{dd}, 11,0,5,5, \mathrm{H}_{\mathrm{b}}-2\right), 4,15(1 \mathrm{H}, \mathrm{dd}, 11,5$, 5,5, H-3), 3,75 (3H, s, 2'-OCH $\left.{ }_{3}\right), 3,72\left(3 \mathrm{H}, \mathrm{s}, 4\right.$ '- $\left.\mathrm{OCH}_{3}\right)$.
${ }^{13} \mathrm{C}-\mathrm{NMR}\left(125 \mathrm{MHz}, \mathrm{DMSO}-\mathrm{d}_{6}\right): 190,3$ (s, C-4), 164,3 (s, C-7), 163,3 (s, C-8a), 158,1 (s, C-4'), 55,2 (s, C-2'), 132.0 (d, C-5), 130,6 (d, C-6'), 116,1 (s, C-1'), 110,5 (d, C-3'), 102,5 (d, C-5'), 104,9 (d, C-6), 114,0 (s, C-4a), 98,8 (d, C-8), 70,3 (t, C-2), 55,6 (q, 4'-OCH ${ }_{3}$ ), 55,2 (q, 2'- $\left.\mathrm{OCH}_{3}\right), 46,6$ (d, C-3).

\subsection{Biện luận xác định cấu trúc hóa học}

Hợp chất (1) được phân lập dưới dạng tinh thể màu nâu. Quan sát trên phổ ${ }^{1} \mathrm{H}-\mathrm{NMR}$ kết hợp với phổ DEPT cho thấy sự hiện diện của 1 nhóm oxymethylene tại $\left[\delta_{\mathrm{H}}\right.$ : $4,52\left(1 \mathrm{H}, \mathrm{t}, 11,0 \mathrm{~Hz}, \mathrm{H}-2_{\mathrm{ax}}\right)$ và 4,4 $(1 \mathrm{H}, \mathrm{dd}, 11,0,5,5$ $\left.\left.\mathrm{Hz}, \mathrm{H}-2_{\mathrm{eq}}\right) / \delta_{\mathrm{C}} 70,3(\mathrm{C}-2)\right], 1$ nhóm methine tại $\left[\delta_{\mathrm{H}} 4,15\right.$ $\left.(1 \mathrm{H}, \mathrm{dd}, 11,5,5,5 \mathrm{~Hz}, \mathrm{H}-3) / \delta_{\mathrm{C}} 46,6(\mathrm{C}-3)\right]$ và 1 nhóm carbonyl tại $\delta_{\mathrm{C}} 190,3$ (C-4). Các tín hiệu này gợi ý đây là một isoflavanone [9].

Các tín hiệu trên phổ ${ }^{1} \mathrm{H}-\mathrm{NMR}$ của hợp chất này đặc trưng cho vòng benzene thế ở các vị trí 1,2,4 (vòng $\mathrm{A}$ và $\mathrm{B})$ bởi các tương tác của 2 hệ spin $\mathrm{ABX}$ tại $\left[\delta_{\mathrm{H}} 7,67\right.$ $(1 \mathrm{H}, \mathrm{d}, \mathrm{J}=9,0 \mathrm{~Hz}, \mathrm{H}-5), 6,54(1 \mathrm{H}, \mathrm{dd}, \mathrm{J}=8,5,2,0 \mathrm{~Hz}$, $\mathrm{H}-6)$ và ở vị trí $6,57(1 \mathrm{H}, \mathrm{d}, \mathrm{J}=2,0 \mathrm{~Hz}, \mathrm{H}-8)$; tại $\delta_{\mathrm{H}}$ : 6,97 (1H, d, 8,5 Hz, H-6'), 6,48 (1H, dd, 9,0, 2,5 Hz, H$\left.5^{\prime}\right)$ và $\left.6,34\left(1 \mathrm{H}, \mathrm{d}, 2,0 \mathrm{~Hz}, \mathrm{H}-3^{\prime}\right)\right]$. Thêm vào đó là tín hiệu của 2 nhóm methoxy tại $\delta_{\mathrm{H}} 3,75\left(3 \mathrm{H}, \mathrm{s}, 2\right.$ '- $\left.-\mathrm{OCH}_{3}\right)$ và $3,72\left(3 \mathrm{H}, \mathrm{s}, 4\right.$ '- $\left.\mathrm{OCH}_{3}\right)$.
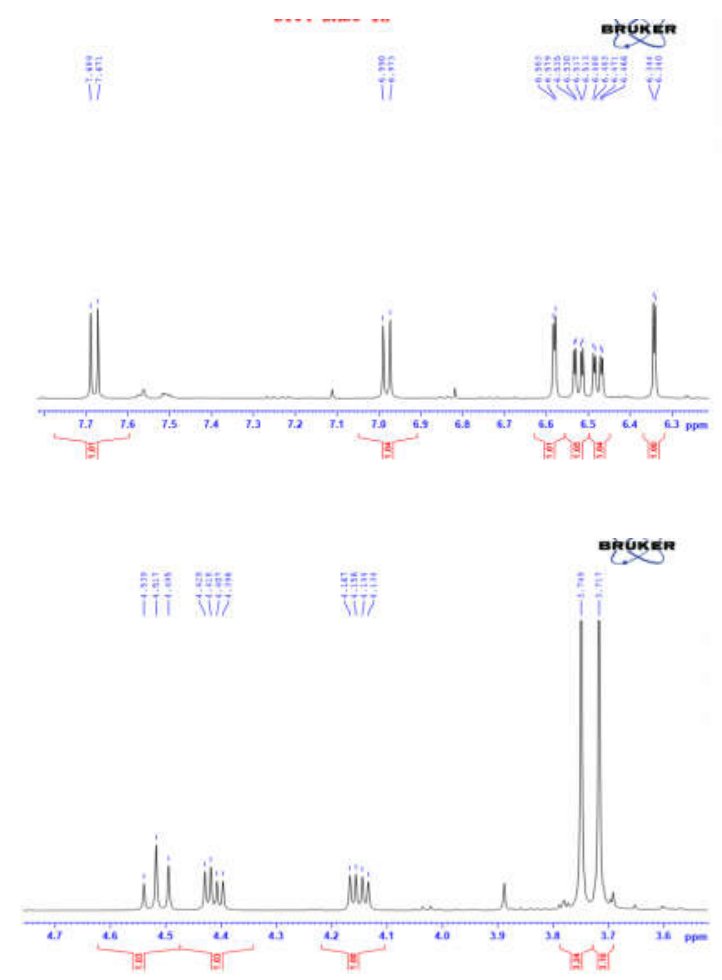

Hình 3. Phổ ${ }^{1}$ H-NMR của hợp chất (1) 


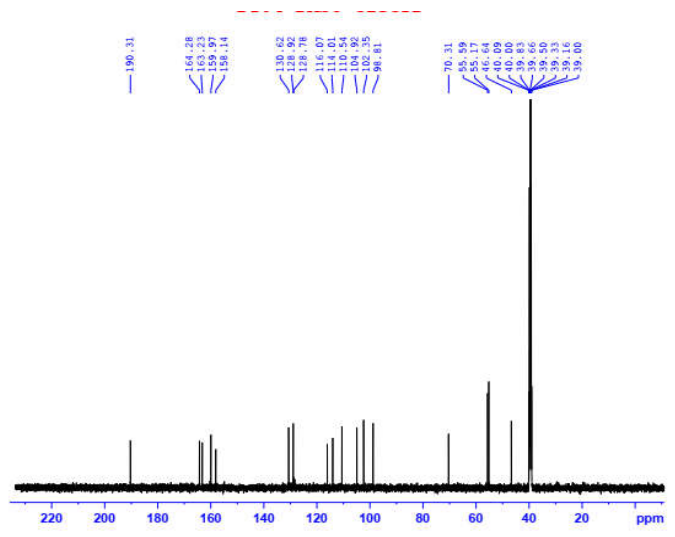

Hình 4. Phổ ${ }^{13}$ C-NMR của hợp chất (1) DEPT90
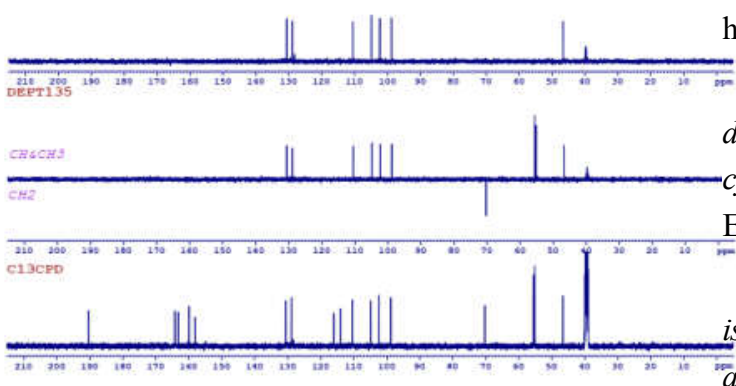

Hình 5. Phổ DEPT của hợp chất (1)

Phổ ${ }^{13} \mathrm{C}$-NMR và DEPT cho thấy tín hiệu của 6 carbon thơm bậc bốn tại các vị trí $\delta_{\mathrm{C}} 164,3$ (C-7), 163,2 (C-8a), 158,1 (C-4'), 159,9 (C-2'), 116,1 (C-1'), 114,0 (C-10), 6 carbon methin thơm tại $\delta_{\mathrm{C}} 130,6$ (C-5), 128,8 (C-6'), 104,9 (C-6), 105,3 (C-5'), 110,5 (C-3'), 98,8 (C-8), 2 nhóm methoxy tại $\delta_{\mathrm{C}} 55,6\left(4^{\prime}-\mathrm{OCH}_{3}\right)$ và $\delta_{\mathrm{C}} 55,2\left(2^{\prime}-\right.$ $\mathrm{OCH}_{3}$ ).

Dựa trên những bằng chứng phổ $\left({ }^{1} \mathrm{H}-\mathrm{NMR},{ }^{13} \mathrm{C}\right.$ NMR, DEPT) phân tích trên cùng với tài liệu tham khảo đã công bố [14]. Chúng tôi đã xác định hợp chất này là một isoflavanone và có tên là sativanone, đã được phân lập từ gỗ loài $D$. parviflora và $D$. odorifera [10]. Sativanone có hoạt tính kháng khuẩn và kháng oxi hóa.

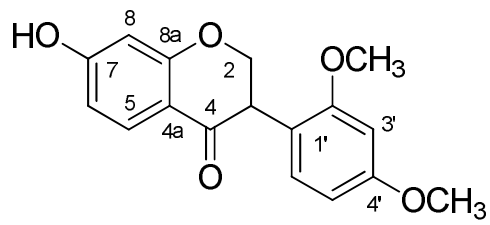

Hình 6. Công thức cấu tạo của (1)

\section{Kết luận}

Bằng các phương pháp sắc kí kết hợp, từ gỗ cây sưa đỏ, hợp chất dạng khung isoflavanone là sativanone đã được phân lập và xác định cấu trúc hóa học bằng phương pháp phân tích hóa lý hiện đại (phổ cộng hưởng từ hạt nhân một chiều và hai chiều), đồng thời kết hợp so sánh với các tài liệu tham khảo. Lần đầu tiên hợp chất này được báo cáo có mặt từ gỗ loài sưa đỏ.

\section{TÀI LIỆU THAM KHẢO}

1. Nguyễn Đăng Khôi (Nguyễn Tiến Bân- chủ biên), Chi Dalbergia L. f. (họ Fabaceae). Danh muc các loài thực vật Việt Nam, Tập II, NXB Nông nghiệp, 2003, 779-786.

2. Đỗ Xuân Cẩm (2013). Cây Sưa ở Huế và các loài Sua ở Việt nam, Tạp chí nghiên cứu và phát triển, 1(99), Tr. 95-100.

3. Trần Ngọc Hải, Bảo tồn và phát triển loài quý hiếm Sua (Dalbergia tonkinensis Prain). Hội nghị khoa học toàn quốc về sinh thái và tài nguyên sinh vật lần thứ hai, Hà Nội, 2010, 34-36.

4. S.-M. Yu, Z.-J. Cheng, S.-C. Kuo, Endotheliumdependent relaxation of rat aorta by butein, a novel cyclic AMP-specific phosphodiesterase inhibitor, European Journal of Pharmacology, 1995, 280, 69-77.

5. S.-M. Yu, S.-C. Kuo, Vasorelaxant effect of isoliquiritigenin, a novel soluble guanylatecyclase activator, in rat aorta, British Journal of Pharmacology, 1995, 114, 1587-1594.

6. Ngu Truong Nhan, Ninh The Son, To Dao Cuong, Nguyen Phuong Dai Nguyen, Pham Ngoc Khanh, Tran Thu Huong, Nguyen Manh Cuong, Further study on chemical constituents from the heartwood of Dalbergia tonkinensis, Vietnam Journal of Science and Technology, 2018, 56 (4A), 252-258 (ACI).

7. Nguyễn Mạnh Cường, Ngũ Trường Nhân, Nguyễn Phương Đại Nguyên, Phạm Ngọc Khanh, Ninh Thế Sơn, Trần Thu Hường, Tô Đạo Cường, Nguyễn Thị Hồng Mai, Nguyễn Thị Phương Trang, So sánh khả năng phân loại loài sua đỏ (Dalbergia tonkinensis) Việt Nam của một số vùng gen luc lạp, Hội nghi Khoa học Công nghệ Sinh học toàn Quốc, Hà Nội, NXB Khoa học Tự nhiên và Công nghệ, 2018, 100-106, ISBN: 978-604-913-759-4.

8. Trần Anh Tuấn, Nguyễn Tiến Đạt, Nguyễn Hoài Nam, Nguyễn Quang Hưng, Trần Minh Hợi, Trần Huy Thái, Châu Văn Minh, Phan Văn Kiệm, Các hợp chất isoflavon và dihydrophenanthren tù̀ cây Sira Bắc Bộ (Dalbergia tonkinensis), Tạp chí Hóa học, 2009, 47 (6), 716-719.

9. P.K. Agrawal, -Carbon 13 NMR of Flavonoids, Studies in Organic Chemistry 39, Amsterdam-OxfordNewTork-Tokyo, 1989, pp 189-191. 
10. X. Zhao, W. Mei, M. Gong, W. Zuo, H. Bai, H. Dalbergia odorifera on Ralstonia solanacearum”, Dai, "Antibacterial Activity of the Flavonoids from Molecules, 2011,16,9775-9782

\section{Studying chemical components of the Dalbergia tonkinesis in Tay Nguyen}

Ngu Truong Nhan, Nguyen Manh Cuong

\section{Article info}

Recieved:

16/02/2020

Accepted:

$10 / 3 / 2020$

Keywords:

Dalbergia tonkinensis;

Fabaceae; isoflavanone;

sativanone; wood;

isolated. 28

\begin{abstract}
From the wood of Dalbergia tonkinensis Prain collected in DakLak province, We used thin-column chromatography and column chromatography methods. The isoflavanone compound is sativanone isolated. The chemical structure of this compound is determined by the magnetic resonance spectrum of NMR nucleus $(1 \mathrm{H}-$ NMR, 13C-; DEPT-NMR spectra), and collated with published references.
\end{abstract}

